\title{
Kaon semileptonic decay form factors with HISQ valence quarks
}

\author{
E. Gámiz ${ }^{* a}$, Jon A. Bailey ${ }^{b}$, A. Bazavov ${ }^{c}$, C. Bernard ${ }^{d}$, C. Bouchard ${ }^{e}$, C. DeTar ${ }^{f}$, \\ D. Du ${ }^{g}$, A.X. El-Khadra ${ }^{g}$, J. Foley ${ }^{f}$, E.D. Freeland ${ }^{h}$, Steven Gottlieb ${ }^{i}$, U.M. Heller ${ }^{j}$, \\ J. Kim $^{k}$, A.S. Kronfeld ${ }^{l}$, J. Laiho ${ }^{m}$, L. Levkova ${ }^{f}$, P.B. Mackenzie ${ }^{l}$, E.T.Neil ${ }^{l}$, \\ M.B. Oktay ${ }^{f}$, Si-Wei Qiu ${ }^{f}$, J.N. Simone ${ }^{l}$, R. Sugar ${ }^{n}$, D. Toussaint ${ }^{k}$, \\ R.S. Van de Water ${ }^{c, l}$, and Ran Zhou ${ }^{i}$ \\ ${ }^{a}$ CAFPE and Departamento de Física Teórica y del Cosmos, Universidad de Granada, Granada, \\ Spain \\ ${ }^{b}$ Department of Physics and Astronomy, Seoul National University, Seoul, South Korea \\ ${ }^{c}$ Physics Department, Brookhaven National Laboratory, ${ }^{\dagger}$ Upton, NY, USA \\ ${ }^{d}$ Department of Physics, Washington University, St. Louis, MO, USA \\ ${ }^{e}$ Department of Physics, The Ohio State University, Columbus, Ohio, USA \\ ${ }^{f}$ Physics Department, University of Utah, Salt Lake City, UT, USA \\ ${ }^{g}$ Physics Department, University of Illinois, Urbana, IL, USA \\ ${ }^{h}$ Department of Physics, Benedictine University, Lisle, Illinois, USA \\ ${ }^{i}$ Department of Physics, Indiana University, Bloomington, IN, USA \\ ${ }^{j}$ American Physical Society, One Research Road, Ridge, NY, USA \\ ${ }^{k}$ Department of Physics, University of Arizona, Tucson, AZ, USA \\ ${ }^{l}$ Fermi National Accelerator Laboratory, ${ }^{\ddagger}$ Batavia, IL, USA \\ ${ }^{m}$ SUPA, School of Physics \& Astronomy, University of Glasgow, Glasgow, UK \\ ${ }^{n}$ Department of Physics, University of California, Santa Barbara, CA, USA \\ E-mail: megamiz@ugr.es
}

\section{Fermilab Lattice and MILC Collaborations}

\begin{abstract}
We report on the status of our kaon semileptonic form factor calculations using the highlyimproved staggered quark (HISQ) formulation to simulate the valence fermions. We present results for the form factor $f_{+}^{K \pi}(0)$ on the asqtad $N_{f}=2+1$ MILC configurations, discuss the chiral-continuum extrapolation, and give a preliminary estimate of the total error. We also present a more preliminary set of results for the same form factor but with the sea quarks also simulated with the HISQ action; these results include data at the physical light quark masses. The improvements that we expect to achieve with the use of the HISQ configurations and simulations at the physical quark masses are briefly discussed.
\end{abstract}

The 30 International Symposium on Lattice Field Theory - Lattice 2012,

June 24-29, 2012

Cairns, Australia 


\section{Introduction and methodology}

The study of exclusive semileptonic decays of $D$ and $K$ mesons provides a way of extracting the Cabibbo-Kobayashi-Maskawa (CKM) matrix elements $\left|V_{c d(c s)}\right|$ and $\left|V_{u s}\right|$ with errors competitive with those obtained using other methods such as leptonic decays, neutrino-antineutrino interactions, or $\tau$ decays. Comparison of the values obtained with different methods could reveal new physics (NP) effects, and comparison of the shape of the form factors describing those exclusive decays with experiment can provide a check of the lattice methodology employed.

Our program includes analyzing $K \rightarrow \pi l v$ and $D \rightarrow K(\pi) l v$ semileptonic decays at zero as well as non-zero momentum transfer. In these proceedings we focus on the status of the $K \rightarrow \pi l v$ analysis at zero momentum transfer, which one can combine with experimental data to extract the CKM matrix element $\left|V_{u s}\right|$. The limiting error currently comes from the lattice determination of the form factors defined in (1.1) [1]. A precise determination of $\left|V_{u s}\right|$ provides stringent tests of first-row unitarity and gives information about the scale of new physics [2].

The theory input needed to extract the CKM matrix elements from exclusive semileptonic widths are form factors parametrizing the corresponding hadronic matrix elements:

$$
\left\langle P_{2}\left|V^{\mu}\right| P_{1}\right\rangle=f_{+}^{P_{1} P_{2}}\left(q^{2}\right)\left(p_{P_{1}}+p_{P_{2}}-\Delta\right)^{\mu}+f_{0}^{P_{1} P_{2}}\left(q^{2}\right) \Delta^{\mu},
$$

where $\Delta^{\mu}=\left(m_{P_{1}}^{2}-m_{P_{2}}^{2}\right) q^{\mu} / q^{2}, q=p_{P_{1}}-p_{P_{2}}$, and $V$ is the appropriate flavor-changing vector current. We obtain the needed form factor $f_{+}^{K \pi}(0)$ using the relation $f_{0}^{K \pi}\left(q^{2}\right)=\frac{m_{s}-m_{l}}{m_{K}^{2}-m_{\pi}^{2}}\langle\pi|S| K\rangle\left(q^{2}\right)$, and the fact that $f_{+}(0)=f_{0}(0)$ due to the kinematic constraint. This method [3] allows us to eliminate the need for a renormalization factor and to extract the form factor from three-point correlation functions with insertion of a scalar current instead of a vector current.

The momentum transfer of the three-point functions is tuned to zero or very close to zero using twisted boundary conditions to inject external momentum [4]. The general structure of the correlation functions is given in Fig. 1. We consider either a moving $\pi\left(\theta_{0}=\theta_{1}=0\right.$ and $\left.\theta_{2} \neq 0\right)$ or a moving $K\left(\theta_{0}=\theta_{2}=0\right.$ and $\left.\theta_{1} \neq 0\right)$, for $K \rightarrow \pi l v$.

\section{HISQ valence fermions and asqtad $N_{f}=2+1$ configurations}

For the first analysis we use the asqtad $N_{f}=2+1$ MILC configurations and the HISQ action to simulate the valence quarks. The strange valence-quark mass is fixed to its physical value and the light valence-quark mass is fixed so $\frac{m_{l}^{\text {val }}(\mathrm{HISQ})}{m_{s}^{\text {hyy }}(\mathrm{HISQ})}=\frac{m_{l}^{\text {sea }}(\text { asqtad })}{m_{s}^{\text {phys }}(\text { asqtad })}$. The parameters of the ensembles included in this calculation and the details of the simulations we perform are collected in Table 1.

In order to extract the value of the form factor $f_{+}^{K \pi}(0)=f_{0}^{K \pi}(0)$, we perform a simultaneous fit of the relevant three- and two-point functions. The correlator fits and the multiple checks performed on their stability under the change of parameters, time range, number of exponentials in the fitting functions, and correlators included, was described in last year's conference proceedings [5]. The

\footnotetext{
*Speaker.

$\dagger$ Operated by Brookhaven Science Associates, LLC, under Contract No. DE-AC02-98CH10886 with the United States Department of Energy.

$¥$ Operated by Fermi Research Alliance, LLC, under Contract No. DE-AC02-07CH11359 with the United States Department of Energy.
} 


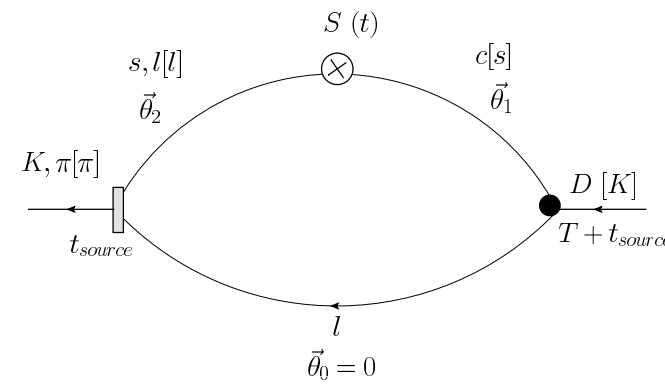

Figure 1: Structure of the 3-point functions needed to calculate $f_{0}^{D K(\pi)}\left[f_{0}^{K \pi}\right]$. Light quark propagators are generated at $t_{\text {source }}$ with random wall sources. An extended charm [strange] propagator is generated at $T+t_{\text {source }}$.

\begin{tabular}{ccccc}
\hline \hline$\approx a(\mathrm{fm})$ & $a m_{l} / a m_{h}$ & $N_{\text {conf }}$ & $N_{\text {sources }}$ & $N_{T}$ \\
\hline 0.12 & $0.020 / 0.050$ & 2052 & 4 & 5 \\
& $0.010 / 0.050$ & 2243 & 4 & 8 \\
& $0.007 / 0.050$ & 2109 & 4 & 5 \\
& $0.005 / 0.050$ & 2098 & 8 & 5 \\
\hline 0.09 & $0.0124 / 0.031$ & 1996 & 4 & 5 \\
& $0.0062 / 0.031$ & 1946 & 4 & 5 \\
\hline \hline
\end{tabular}

Table 1: Aqstad nsembles and simulation details. $a m_{h}$ is the nominal strange-quark mass in the sea sector, $N_{\text {sources }}$ is the number of time sources, and $N_{T}$ the number of sinksource separations.

conclusion is that we find it very difficult to make changes in the fitting procedure that change the fit results outside the one sigma range. The only change with respect to last year's fits is that in our final correlator fits we are including functions with the momentum injected in both the $\pi$ and in the $K$. The results from these combined fits for the different ensembles are shown in Fig. 2. Statistical errors are very small, $\sim 0.1-0.15 \%$.

\subsection{Chiral-continuum extrapolations}

We need to extrapolate our results to the continuum limit and the physical light-quark masses, and also adjust for the mistuning of the strange sea-quark mass in the asqtad $N_{f}=2+1$ MILC configurations. Our plan is to perform these extrapolations using partially quenched staggered chiral perturbation theory $(\mathrm{S} \chi \mathrm{PT})$ at NLO plus regular continuum $\chi \mathrm{PT}$ at NNLO. Addressing staggered effects at NLO should be enough to achieve the sub-percent precision that we target.

In the continuum, the form factor is given by $f_{+}^{K \pi}(0)=1+f_{2}+f_{4}+f_{6}+\ldots$, where, according to the Ademollo-Gatto (AG) theorem, the chiral corrections $f_{2}, f_{4}, f_{6} \ldots$ go to zero in the $S U(3)$ limit as $\left(m_{K}^{2}-m_{\pi}^{2}\right)^{2}$. This means that at NLO there are no free low-energy constants and $f_{2}$ is fixed in terms of experimental quantities. At finite lattice spacing, however, we would have violations of the AG theorem due to discretization effects in the continuum dispersion relation needed to derive the relation between $f_{0}^{K \pi}(0)$ and the correlation functions we are generating.

The general structure of the fitting function we plan to use for our chiral fits is thus

$$
\begin{aligned}
f_{+}^{K \pi}(0)=1+f_{2}^{P Q, \text { stag. }}(a)+ & C_{4}^{(a)}\left(\frac{a}{r_{1}}\right)^{2}+f_{4}^{\text {cont. }}(\operatorname{logs})+f_{4}^{\text {cont. }}\left(L_{i}\right) \\
& +r_{1}^{4}\left(m_{\pi}^{2}-m_{K}^{2}\right)^{2}\left[C_{6}^{(1)}+C_{6}^{(a)}\left(\frac{a}{r_{1}}\right)^{2}\right],
\end{aligned}
$$

where the constants $C_{4}^{(a)}, C_{6}^{(a)}$, and $C_{6}^{\prime(1)} \propto C_{12}+C_{34}-L_{5}^{2}$ are free parameters to be fixed by the chiral fits. The $L_{i}^{\prime} s$ are the usual $\mathscr{O}\left(p^{4}\right)$ low-energy constants (LEC's), and the $C_{i j}$ are $\mathscr{O}\left(p^{6}\right)$ LEC's defined in [7]. The function $f_{2}^{P Q, \text { stag. }}(a)$ is the NLO partially quenched $\mathrm{S} \chi \mathrm{PT}$ expression, which incorporates the dominant lattice artifacts from taste breaking. To a very good approximation, there are no free parameters in that function. The taste-splitting and the taste-violating hairpin parameters 
are already available for both the valence and the sea quarks from lattice calculations for asqtad or HISQ fermions, respectively. We are also in the process of calculating the taste-splittings for the mixed mesons, those made of one sea fermion and one valence fermion. The only parameter that we do not get from other calculations is an extra taste-violating hairpin parameter that appears due to the fact that we have a mixed action. We need to leave this quantity as a free parameter of the fit, although we expect its impact to be small. We take the NNLO contribution, $f_{4}$, from the calculation in [6]. Finally, we try to include terms proportional to $a^{2}$ with free parameters $C_{4}^{(a)}$ (to take into account the violations of the $\mathrm{AG}$ theorem at finite lattice spacing) and $C_{6}^{(a)}$ (to account for the residual $a^{2}$ dependence at higher orders).

Since we have not completely checked the NLO partially quenched S $\chi$ PT calculation, here we use a simplified version without hairpin terms. We also approximate the taste-splittings of the mixed action mesons by the average of the sea and the valence values, $\Delta_{\text {mix }}=\left(\Delta_{\text {sea }}(\right.$ asqtad $)+$ $\left.\Delta_{\text {valence }}(\mathrm{HISQ})\right) / 2$ (we checked that using the preliminary values for the correct splitting does not change the extrapolated $f_{+}^{K \pi}$ by more than $0.1 \%$ ). With these simplifications, we tried several variations of the fitting function in (2.1): fixing the LEC's $L_{i}$ 's to their value from the global fit in [8], fixing them to the value from the fits in [9], or leaving the $L_{i}$ 's as free parameters in the fit with prior central values equal to the results in [8] and varying the prior widths from the errors in [8] to an order of magnitude larger; including only the term proportional to $C_{4}^{(a)}$, the one proportional to $C_{6}^{(a)}$, or both, etc. The extrapolated value for $f_{+}^{K \pi}(0)$ has statistical errors between $0.2 \%$ and $0.3 \%$ in all cases and the different results agree with each other within one statistical $\sigma$. The violations of the AG theorem are around $0.32-0.15 \%$ for $a \approx 0.12 \mathrm{fm}$ and $0.15-0.1 \%$ for $a \approx 0.09 \mathrm{fm}$. A typical example of the fits we have performed is shown in the left side of Fig. 2.

In order to check the impact of the choice of fitting function in the extrapolation, we have also done a number of fits replacing the NNLO continuum $\chi \mathrm{PT}$ functions by a NNLO analytical parametrization

$$
\begin{aligned}
f_{+}^{K \pi}(0)=1 & +f_{2}^{P Q, \text { stag. }}+C_{4}^{(a)}\left(\frac{a}{r_{1}}\right)^{2}+r_{1}^{4}\left(m_{\pi}^{2}-m_{K}^{2}\right)^{2}\left[C_{6}^{(1)}\left(r_{1} m_{\pi}\right)^{2}+C_{6}^{(2)}\left(r_{1} m_{K}\right)^{2}\right. \\
& \left.+C_{6}^{(3)}\left(r_{1} m_{\pi}\right)^{2} \ln \left(m_{\pi}^{2} / \mu^{2}\right)+C_{6}^{(4)}\left(r_{1} m_{\pi}\right)^{4}+C_{6}^{(a)}\left(\frac{a}{r_{1}}\right)^{2}\right]
\end{aligned}
$$

where $f_{2}^{P Q, \text { stag. }}$ is the same partially quenched NLO S $\chi \mathrm{PT}$ expression as in (2.1) and the $C_{4}^{(a)}, C_{6}^{(a)}$, and $C_{6}^{(i)}$ with $i=1-4$ are the free parameters in the fit. Again, we tried variations of the functional form in (2.2), turning on and off different NNLO and $a^{2}$ terms, and parametrizing them in different ways. All the fitting functions we tried in this category gave results within one statistical $\sigma$ of each other. In the right-hand side of Fig. 2 we plot an example of these fits. Both methods to describe NNLO contributions give results that, again, agree within one statistical $\sigma$.

\section{Preliminary results with HISQ valence quarks and HISQ $\mathbf{N}_{\mathrm{f}}=2+1+1$ configurations}

The second stage of our semileptonic decay program is the study of decays with $q^{2}=0$ for $D$ and $K$ mesons using HISQ for the valence and the sea quarks, i.e., simulating on the HISQ $N_{f}=2+1+1$ MILC configurations. 

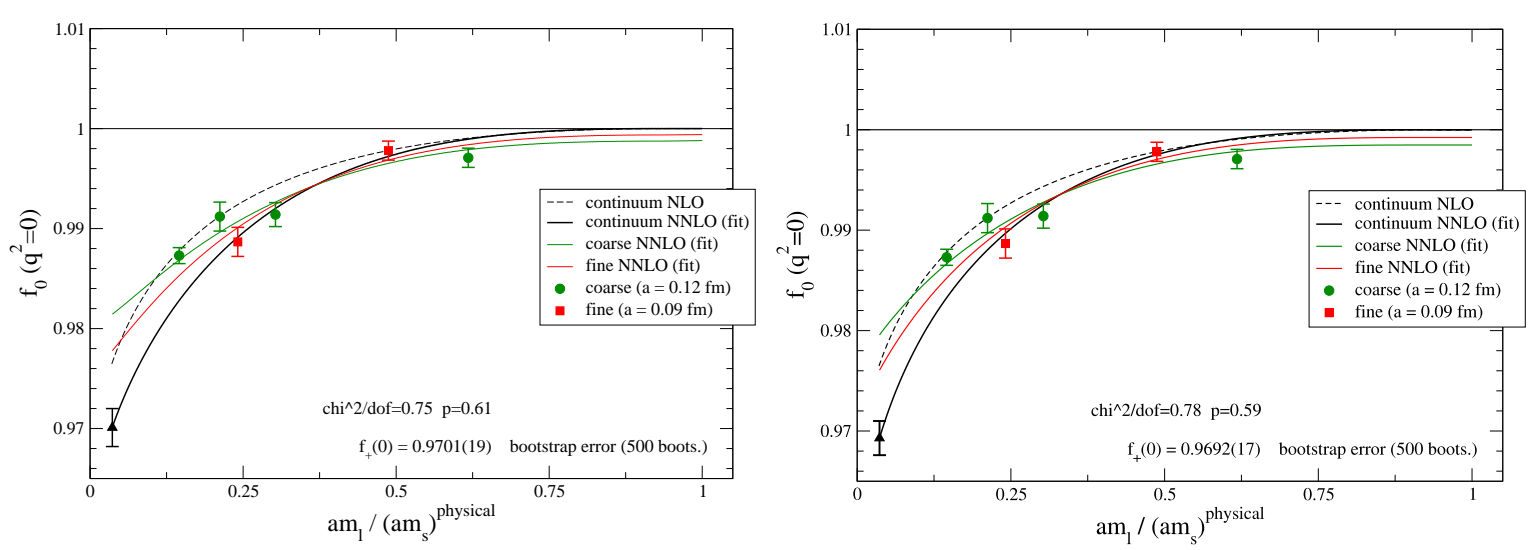

Figure 2: Left-hand plot: example of chiral-continuum extrapolations using the fit function in Eq. (2.1) with $C_{6}^{(a)}=0$ and free $L_{i}$ 's and priors equal to the results in Ref. [8]. Right-hand side: example using the fit function in Eq. (2.2) with $C_{6}^{(a)}=C_{6}^{(3)}=C_{6}^{(4)}=0$. Errors are statistical only, from 500 bootstrap ensembles.

The setup and methodology of the calculation is common to the asqtad $N_{f}=2+1$ calculation and is described in Sec. 1. Aside from the reduction in discretization errors provided by having HISQ sea quarks, the main improvement of this calculation respect to the asqtad $N_{f}=2+1$ one is that we will include ensembles with physical light-quark masses. The parameters of the ensembles we plan to include in this analysis, as well as the status of the runs, are shown in Table 2. The plan is to have around 1000 configurations per ensemble and 4-5 different source-sink separations per ensemble at three different values of the lattice spacing. For the key ensembles we are generating data for 8 time sources, and, for the remaining ensembles, 4 time sources. For the light and strange valence-quark masses we will use the physical values. For the charm-quark masses, we will simulate at a value equal to the sea charm-quark mass in addition to the current estimate of the physical one, to allow for later corrections of $a m_{c}^{\text {phys }}$.

\begin{tabular}{ccccrccc}
\hline \hline$\approx a(\mathrm{fm})$ & $a m_{l} / a m_{h}$ & $a m_{c}$ & Volume & $N_{\text {conf }}$ available & $N_{\text {sources }}$ & $N_{T}$ & $\%$ run completed \\
\hline 0.15 & 0.035 & 0.831 & $32^{3} \times 48$ & 1020 & 8 & 5 & 100 \\
\hline 0.12 & 0.200 & 0.635 & $24^{3} \times 64$ & 1053 & 4 & 4 & 100 \\
& 0.100 & 0.628 & $32^{3} \times 64$ & 1020 & 4 & 4 & 0 \\
& 0.035 & 0.628 & $48^{3} \times 64$ & 460 & 8 & 4 & 50 \\
\hline 0.09 & 0.200 & 0.440 & $32^{3} \times 96$ & 1011 & 4 & 4 & 0 \\
& 0.100 & 0.430 & $48^{3} \times 96$ & 1000 & 4 & 4 & 0 \\
& 0.035 & 0.432 & $64^{3} \times 96$ & 497 & 8 & 4 & 0 \\
\hline \hline
\end{tabular}

Table 2: HISQ ensembles and simulation details. $N_{\text {sources }}$ is the number of time sources, and $N_{T}$ the number of sink-source separations for which we have generated data. The number of configurations, $N_{\text {conf }}$ available and the status of the runs (last column) correspond to July 2012.

In these runs we generate the correlation functions needed for the calculation of the form factor at zero momentum transfer for both $K \rightarrow \pi l v$ and $D \rightarrow K(\pi) l v$. The energies of the pions 


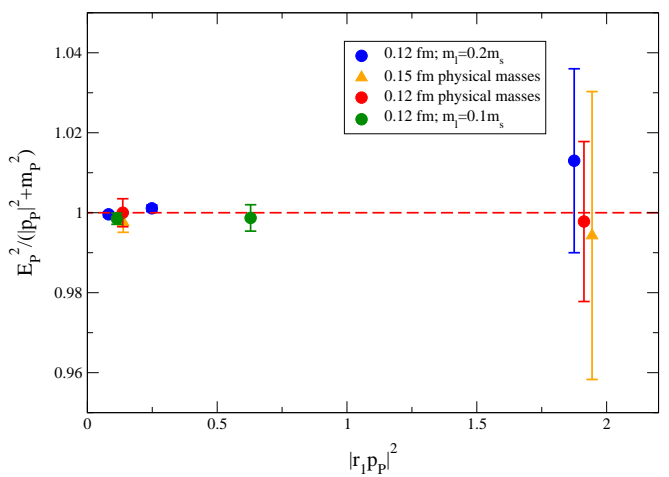

Figure 3: Deviation of our data from the continuum dispersion relation prediction.

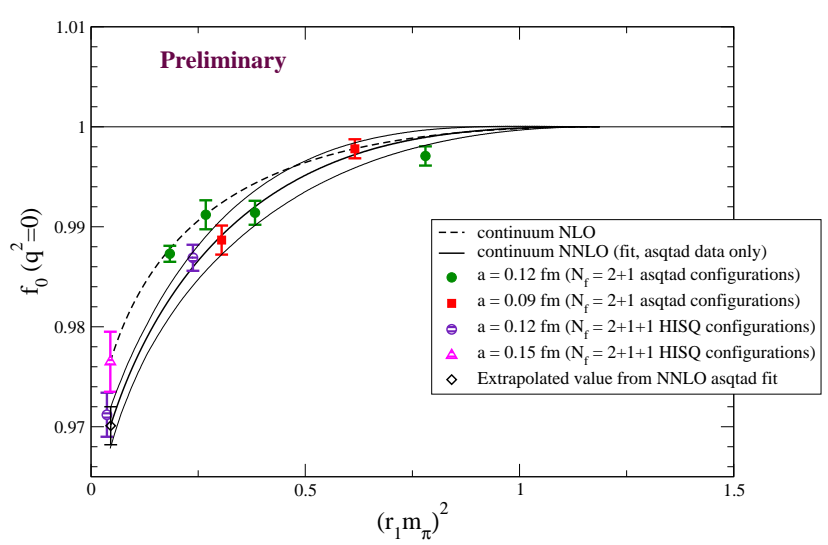

Figure 4: Form factor $f_{+}^{K \pi}(0)$ as a function of the $\pi$ mass from the asqtad $N_{f}=2+1$ and the HISQ $N_{f}=2+1+1$ calculations, together with the results from a fit to asqtad $N_{f}=2+1$ alone, also plotted on the left-hand side of Fig. 2.

and kaons generated on those ensembles show very little deviation from the continuum dispersion relation, see Fig. 3. The points with larger errors in that plot correspond to the energies needed to inject momentum in a $K$ to get $q^{2}=0$ in $K \rightarrow \pi l v$ when we have physical light-quark masses. Thus, for physical light-quark masses, moving pions will give us significantly smaller statistical errors than moving kaons in $K \rightarrow \pi l v$ decays.

We fit the correlation functions generated on the HISQ configurations for the $K \rightarrow \pi l v$ decays using the same fitting functions and following the same strategy described in Sec. 1. The preliminary results from these fits are shown in Fig. 4, together with the data generated on the asqtad configurations and the results from the fit to the asqtad data alone (left plot in Fig. 2). The $N_{f}=2+1+1$ HISQ data are very close to the continuum line obtained by fitting the $N_{f}=2+1$ asqtad data. In particular, the point corresponding to the ensemble with physical quark masses and $a \approx 0.12 \mathrm{fm}$ lies right on top of the extrapolated value we got from the asqtad fit. This seems to indicate that the discretization effects in the HISQ data are going to be smaller than in the asqtad data, as expected. The statistical error of the physical mass point is $\sim 0.2 \%$, larger than the $0.1-0.15 \%$ error we got for larger masses, but of the same order as the extrapolated value.

\section{Conclusions}

We have nearly completed the calculation of $f_{+}^{K \pi}(0)$ at two different values of the lattice spacing using the asqtad $N_{f}=2+1$ MILC configurations. The last step towards finishing the calculation is checking the NLO partially quenched $\mathrm{S} \chi \mathrm{PT}$ expressions and completing the error budget. We estimate that the total error is going to be between $0.35-0.5 \%$, dominated by the statistical and extrapolation errors $(0.2-0.3 \%)$ and the uncertainty associated with the deviation of $a m_{s}^{\text {sea }}$ from the physical value $(\sim 0.2 \%)$. We are also investigating the impact of subleading errors, such as finite volume effects. The total error will be competitive with current state-of-the-art calculations $[10,11]$. 
The dominant two errors in the calculation on the asqtad $N_{f}=2+1$ configurations will be reduced in the next step of our program namely, the calculation on the HISQ $N_{f}=2+1+1$ configurations, for which we have shown preliminary results here. Having data at the physical quark masses will reduce the statistical and extrapolation errors, as well as the one associated with the choice of chiral fitting function. Discretization errors are also considerably smaller for the HISQ action than for the asqtad action, as explicitly seen in Fig. 4. Finally, the strange sea-quark masses are much better tuned on the HISQ ensembles, and we are including the effects of the dynamical charm-quark.

\section{Acknowledgments}

We thank Johan Bijnens for making his NLO partially quenched $\chi$ PT and NNLO full QCD $\chi$ PT codes available to us. Computations for this work were carried out with resources provided by the USQCD Collaboration and the Argonne Leadership Computing Facility, the National Energy Research Scientific Computing Center, and the Los Alamos National Laboratory, which are funded by the Office of Science of the U.S. Department of Energy; and with resources provided by the National Institute for Computational Science, the Pittsburgh Supercomputer Center, the San Diego Supercomputer Center, and the Texas Advanced Computing Center, which are funded through the National Science Foundation's Teragrid/XSEDE Program. This work was supported in part by the MICINN (Spain) under grant FPA2010-16696 and Ramón y Cajal program (E.G.), Junta de Andalucía (Spain) under grants FQM-101, FQM-330, and FQM-6552 (E.G.), European Commission under Grant No. PCIG10-GA-2011-303781 (E.G.), by the U.S. Department of Energy under Grant No. DE-FG02-91ER40677 (A.X.E.) and DE-FG02-91ER40628 (C.W.B.), and by the U.S. National Science Foundation under grants PHY0757333 and PHY1067881 (C.D.).

\section{References}

[1] M. Antonelli et al., Eur. Phys. J. C69, 399-424 (2010). [arXiv:1005.2323 [hep-ph]].

[2] V. Cirigliano, J. Jenkins and M. Gonzalez-Alonso, Nucl. Phys. B 830 (2010) 95 [arXiv:0908.1754 [hep-ph]].

[3] H. Na, C. T. H. Davies, E. Follana, G. P. Lepage, J. Shigemitsu, Phys. Rev. D82 (2010) 114506. [arXiv:1008.4562 [hep-lat]].

[4] C. T. Sachrajda and G. Villadoro, Phys. Lett. B 609 (2005) 73 [hep-lat/0411033].

[5] E. Gámiz, C. DeTar, A. X. El-Khadra, A. S. Kronfeld, P. B. Mackenzie and J. Simone, PoS (Lattice 2011) 281 [arXiv:1111.2021 [hep-lat]].

[6] J. Bijnens, P. Talavera, Nucl. Phys. B669 (2003) 341-362. [hep-ph/0303103].

[7] J. Bijnens, G. Colangelo and G. Ecker, JHEP 9902 (1999) 020 [hep-ph/9902437].

[8] G. Amoros, J. Bijnens and P. Talavera, Nucl. Phys. B 602 (2001) 87 [hep-ph/0101127].

[9] A. Bazavov et al. [MILC Collaboration], PoS (Lattice 2009 ) 079 [arXiv:0910.3618 [hep-lat]].

[10] P. A. Boyle et al. [RBC-UKQCD Collaboration], Eur. Phys. J. C 69 (2010) 159 [arXiv:1004.0886 [hep-lat]].

[11] V. Lubicz et al. [ETM Collaboration], Phys. Rev. D 80 (2009) 111502 [arXiv:0906.4728 [hep-lat]]. 